\section{Psychiatric effects of cannabis ${ }^{\dagger}$}

\author{
ANDREW JOHNS
}

\section{UNTOWARD MENTAL EFFECTS OF CANNABIS}

The untoward mental effects of cannabis may be classified:

(a) Psychological responses such as panic, anxiety, depression or psychosis. These effects may be described as 'toxic' in that they generally relate to excess consumption of the drug.

(b) Effects of cannabis on pre-existing mental illness and cannabis as a riskfactor for mental illness.

(c) Dependency or withdrawal effects.

The effects of cannabis on cognition are separately reviewed by Ashton (2001, this issue).

\section{PSYCHOLOGICAL RESPONSES TO CANNABIS}

There is good evidence that taking cannabis leads to acute adverse mental effects in a high proportion of regular users. Many of these effects are dose-related, but adverse symptoms may be aggravated by constitutional factors including youthfulness, personality attributes and vulnerability to serious mental illness.

\section{Cannabis and mood change}

The acute response to cannabis generally includes euphoria and feelings of detachment and relaxation. Adverse effects are not uncommon: these are generally shortlived, but may persist or recur with continued use of the drug.

From New Zealand, a sample of 1000 people aged 18-25 were asked to complete a self-administered questionnaire on cannabis use and related problems (Thomas, 1996). Those respondents who admitted using cannabis (38\%) were asked about mental health consequences; of these, $22 \%$ reported panic attacks or anxiety. Women were twice as likely as men to report these symptoms. Troisi et al (1998) used urine tests on Italian draftees to identify 133 men who used only cannabis. All individuals with a pre-existing psychosis or severe personality disorder had been excluded. An adjustment disorder with depressed mood was found in $16 \%$, major depression in $14 \%$, and dysthymia in $10.5 \%$. The severity of these symptoms was dose-related. No acute psychotic symptoms were reported. Reilly et al (1998) describe the adverse effects found among 268 cannabis users who had taken the drug for at least 10 years, and who continued to smoke about two refers a day. The most common adverse effects were feelings of anxiety, paranoia or depression $(21 \%)$, tiredness and low motivation (21\%).

Among individuals making serious attempts at suicide, $16.2 \%$ met criteria for cannabis misuse/dependence compared with $1.9 \%$ of controls - much of the highly significant association was thought to be due to independent variables including comorbidity, but it is suggested that cannabis misuse makes a direct contribution to the risk of serious self-harm, either directly or by aggravation of other mental disorders (Beautrais et al, 1999).

\section{Cannabis and psychosis}

Cannabis use can lead to a range of shortlived symptoms such as depersonalisation, derealisation, a feeling of loss of control, fear of dying, irrational panic and paranoid ideas (Thomas, 1993). For example, Thomas (1996) reported that, among cannabis users who responded to his survey, $15 \%$ identified psychotic symptoms such as hearing voices or having unwarranted feelings of persecution or risk of harm from others. Two small case studies have reported prolonged depersonalisation after cessation of cannabis use (Szymanski, 1981; Keshaven \& Lishman, 1986). 'Flashbacks' or the subsequent partial re-experience when drug-free of symptoms experienced during intoxication are rarely reported after cannabis use (Thomas, 1993).

The casual use of the term 'cannabis psychosis' in clinical psychiatric practice and in the scientific literature results in diagnostic imprecision and research of uncertain validity. Thornicroft (1990) reviews the possible associations between cannabis use and psychosis and suggests that common methodological failings are: 
(a) studies fail to adequately separate organic from functional psychotic reactions to cannabis; (b) they have insufficiently discriminated between psychotic symptoms and syndromes of a psychosis; and (c) they have not balanced the weight of evidence for and against the category of cannabis psychosis. Although there is good evidence for believing that cannabis use may in certain circumstances contribute to psychotic disorders, the connections are complex.

Hall et al (1994) suggest that the fundamental questions are: is there a cannabis psychosis, and does cannabis precipitate an underlying psychosis? In theory, cannabis use may precipitate a psychosis in the following ways.

(a) Acute use of large doses of the drug may induce a toxic or organic psychosis with symptoms of confusion and hallucination, which remit on abstinence.

(b) Cannabis use may lead to an acute functional psychosis, similar to an acute schizophreniform state and lacking the organic features of a toxic psychosis.

(c) Cannabis use may lead to a chronic psychosis, which persists after abstinence.

(d) Long-term cannabis use may lead to an organic psychosis which only partially remits after abstinence, leaving a residual deficit state, sometimes called an amotivational syndrome, which is thought to be analogous to the chronic organic brain syndrome seen after prolonged misuse of alcohol.

(e) Cannabis use may be a risk-factor for serious mental illness such as schizophrenia.

\section{Cannabis and toxic psychosis}

Apart from single-case reports, the nature of cannabis-induced toxic psychosis is considered in the following studies, all of which are weakened by the lack of urinetesting to confirm the presence of cannabis and the absence of other drugs of misuse.

Talbott \& Teague (1969) described 12 soldiers in Vietnam who, after their first admitted use of cannabis, showed disorientation, impaired memory, confusion, reduced attention span and disordered thinking with labile effect and hallucinations. These symptoms resolved within a week. Tennant \& Groesbeck (1972) describe psychoses among 36000 US servicemen stationed in Germany. Of the
5120 soldiers using cannabis at least three times a week, 720 presented with cannabis-related problems. The hashish available was potent, containing $5-10 \%$ tetrahydrocannabinol (THC). The authors identified 19 cases of a panic attack or short-lived toxic psychosis, which appeared after a single high dose of hashish, and a further 85 cases of toxic psychosis which appeared after the consumption of cannabis with other drugs. These acute states tended to resolve within 3 days.

From Calcutta, Chopra \& Smith (1974) retrospectively identified 200 in-patients who showed serious psychiatric symptoms after taking cannabis. The most common symptoms in all patients were sudden onset of confusion, often associated with hallucinations and emotional lability. Disorientation, depersonalisation and paranoid symptoms were common. Many patients had taken a large dose of cannabis, which was followed by an intoxicated state for which they were subsequently amnesic. Among the $34 \%$ of patients without a previous history of psychiatric disorder, adverse symptoms lasted no more than a few days, followed by full recovery. A previous history of schizophrenia or personality disorder was associated with longer duration of adverse symptoms.

From Pakistan, Chaudry et al (1991) report on effects of bhang, a potent beverage made from an infusion of cannabis leaves and flowering tops. They identified 15 patients who having taken bhang, presented with a psychosis with symptoms of grandiosity, excitement, hostility, disorientation, hallucinations and thought disorder. Mental state was assessed systematically, using the Brief Psychiatric Rating Scale (BPRS) (Lukoff et al, 1986). The control group of 10 patients all used bhang, but less frequently than the study group.

This work suggests that cannabis, especially in high doses, can produce a toxic psychosis in individuals who have no history of severe mental illness. The main features are mild impairment of consciousness, distorted sense of passage of time, dream-like euphoria, progressing to fragmented thought processes and hallucinations, generally resolving within a week of abstinence (Lishman, 1998).

\section{Cannabis and acute functional psychosis}

A number of studies suggest that heavy cannabis use can lead to an acute functional illness, that is a state resembling the psychosis of acute schizophrenia without the amnesia and confusion of a toxic psychosis.

Tennant \& Groesbeck (1972) identified 115 cases of schizophrenic reaction among the 720 regular users of cannabis; however, all but three had used cannabis with other drugs or alcohol. Thacore \& Shukla (1976) compared 25 individuals with a putative diagnosis of 'cannabis psychosis of the paranoid type' with controls diagnosed with paranoid schizophrenia. Patients with cannabis psychosis showed more bizarre behaviour, violence, panicky affect, more insight and less evidence of thought disorder. They also showed a rapid response to neuroleptics with complete recovery. More robust in methodology is the work of Rottanburg et al (1982) in which 20 patients with psychosis and with high urinary cannabinoids were compared with 20 matched cannabis-free controls. Mental state was assessed using the Present State Examination (PSE) (Wing et al, 1974). The cannabis-positive patients had more symptoms of hypomania and agitation, less auditory hallucinations, flattening of affect, incoherent speech and hysteria than controls. Clouding of consciousness was absent in most cannabis patients. They also showed marked improvements in symptoms within a week, while the controls remained unwell despite receiving comparable antipsychotic drugs. The authors conclude that a high intake of cannabis may be related to a rapidly resolving psychosis with marked hypomanic features. However, 16 cannabis-positive psychotic patients left the study prematurely, which may bias the findings on the 20 who remained. Rapid resolution of symptoms is also reported by Carney et al (1984), who identified nine patients with cannabis-related psychotic episodes. Their differing symptomatology was described as 'schizophreniform, manic, delusional psychosis and confusion'.

More recently, Mathers \& Ghodse (1992) carried out a prospective study of in-patients with psychotic symptoms and cannabis-positive urine. Blind to the urine test result, researchers applied the PSE on admission and again at 1 and 6 months. Concurrently admitted patients with psychosis but with drug-free urine analysis were controls. At 1 week the two groups differed significantly on only five PSE items: changed perception, thought insertion, non-verbal auditory hallucinations, delusions of control, and delusions of 
grandiose ability; this symptom cluster at 1 week was thought to be consistent with acute cannabis intoxication. These differences were minor at 1 month and absent at 6 months. Chronic cannabis-induced psychosis was not found. Caucasian patients were more likely to be depressed with depersonalisation and derealisation, while African-Caribbeans showed more culturally influenced delusions. However, these findings could not be replicated by McGuire et al (1994) who also used the PSE to assess the psychopathology of 23 patients with psychosis who were cannabispositive on urinary screening, and 46 matched drug-free controls. Cases and controls were indistinguishable in terms of psychopathology, DSM-III diagnoses (American Psychiatric Association, 1980), onset of recent illness, the proportion of first admissions, ethnicity and socioeconomic class, differing only in their histories of substance use.

Having compared groups of drugmisusing patients with psychosis of varying duration, Tsuang et al (1982) concluded that the shorter-duration disorders were drug-induced toxic psychoses, and the longer-lasting disorders represented the expression of functional psychiatric illness in vulnerable individuals. If corroborated, this suggests that the 'functional psychosis' related to cannabis use is best explained as a precipitated episode of an underlying functional illness.

\section{Cannabis and chronic psychosis}

Ghodse (1986) has suggested that regular heavy users of cannabis may suffer repeated short episodes of psychosis and effectively 'maintain' themselves in a chronic psychotic state. This is a possibility, but Hall et al (1994) note that it is difficult to distinguish between a chronic cannabis psychosis and the co-occurrence of an illness such as schizophrenia with continued cannabis use. There is however, no robust evidence that heavy cannabis use may lead to a psychotic illness which persists after abstinence (Thomas, 1993).

\section{Cannabis and amotivational syndrome}

It has been suggested that heavy cannabis use could lead to an 'amotivational syndrome' described as personality deterioration with loss of energy and drive to work (Tennant \& Groesbeck, 1972). The supporting evidence largely comprises uncontrolled studies of long-term cannabis users in various cultures (Hall et al, 1994). It is probable that amotivational syndrome represents nothing more than ongoing intoxication in frequent users of the drug (Negrete et al, 1986) and the validity of this diagnosis remains uncertain (Hall et al, 1994).

\section{Cannabis as risk-factor for serious mentall illness}

\section{Comorbidity rates}

Cannabis use is associated with high rates of comorbidity for other psychiatric diagnoses. The Epidemiologic Catchment Area (ECA) survey (Regier et al, 1990) of 20000 subjects in community and institutional settings showed that $50.1 \%$ of individuals with cannabis dependence/ misuse also met DSM-III criteria for one other non-drug or alcohol mental disorder. Among 133 Italian draftees, Troisi et al (1998) found that the prevalence of comorbidity was significantly related to the pattern of cannabis use: $69 \%$ of subjects with DSM-III-R cannabis dependence, $41 \%$ of those with cannabis abuse and $24 \%$ of occasional users reported at least one DSM-III-R Axis 1 psychiatric diagnosis. Most common were adjustment disorder with depressed mood $(n=21)$, major depression $(n=19)$ and dysthymia $(n=14)$. The severity of symptoms also increased with degree of cannabis use. Psychotic symptoms were not found, but it should be noted all individuals with psychotic illness or severe personality disorder were not drafted.

There are high rates of drug misuse among people with mental illness. The ECA study (Regier et al, 1990) showed that the risk of meeting criteria for a substance misuse disorder was 4.6 times higher in those suffering from schizophrenia than in the general population. Schizophrenia was associated with a six-fold increase in risk of developing a drug use disorder, and cannabis was the most commonly misused drug. Menezes et al (1996) examined the prevalence of substance misuse problems among 171 patients with psychotic illness who had any contact with mental health treatment services in a south London area. Alcohol problems were more prevalent, but current use of one or more drugs was found in 35 subjects $(20 \%)$; all but two said they used cannabis. Cantwell et al (1999) studied 168 subjects presenting with a first episode of psychosis and found 1-year prevalence rates of $19.5 \%$ for drug misuse, $11.7 \%$ for alcohol misuse, and cannabis was the most commonly misused substance. Given these findings, it is necessary to review the possible role of cannabis as a risk factor for functional illness and for the aggravation of symptoms.

\section{Effects of cannabis on severe mental illness}

Given that high doses of cannabis can cause a toxic psychosis, then it may be supposed it will aggravate the symptoms of schizophrenia. However, clinical experience suggests that some patients say that they take cannabis as a form of 'self-medication'. For example, Dixon et al (1990) interviewed 83 patients with schizophrenia or schizophreniform psychoses who reported that cannabis reduced anxiety and depression, led to increased suspiciousness and had varied effects on drive and hallucinations. Arndt et al (1992) investigated a cohort of 131 patients with schizophrenia and found that previous use of cannabis had no impact on current symptoms. Peralta \& Cuesta (1992) reported that cannabis had no significant effect on positive symptoms of schizophrenia, but it did attenuate negative symptoms.

On the other hand, there are a few controlled studies that have tended to demonstrate that cannabis aggravates the severity of positive symptoms. Negrete et al (1986) described the history of confirmed cannabis use in 137 patients with schizophrenia in treatment. Subjects who were using cannabis over the 6-month observation period presented with significantly greater delusions and hallucinations, and made more use of psychiatric services. Similarly, Cleghorn et al (1991) found that drug-users with schizophrenia, among whom cannabis was the most heavily used drug, had a higher prevalence of hallucinations, delusions and other positive symptoms. This finding was replicated by Baigent et al (1995), who reported that among 53 in-patients with a dual diagnosis of substance misuse and schizophrenia, cannabis was the only drug that worsened positive symptoms.

Data from the ECA survey (Swanson et al, 1990) also casts some light on the possible effects of cannabis use disorder and violence. Subjects were asked about episodes of violence in the previous year (i.e. hitting a partner, bruising a child, fighting, using a weapon in a fight while drinking). Of the 191 respondents with 
cannabis abuse or dependence, $19.25 \%$ (risk ratio 9.4) had been violent compared with $12.69 \%$ (risk ratio 6.2 ) of those with schizophrenia or schizophreniform disorder and $24.57 \%$ (risk ratio 11.9) of those with alcohol abuse or dependence. Here, the risk is expressed relative to the $2.05 \%$ who were violent among those of the sample population who showed no psychiatric disorder. However, this does not amount to a causal correlation between cannabis co-morbidity and violence, given the possible role of intervening variables such as individual and social factors.

That cannabis consumption also has an adverse effect on the course of schizophrenia was noted by Negrete et al (1986) and confirmed in a prospective study by Linszman et al (1994). A cohort of newly admitted patients with schizophrenia were assessed monthly for a year, using the BPRS and self-reports of cannabis use. The cannabis-using group $(n=24)$ experienced significantly more and earlier psychotic relapses and this effect was dose-related.

As Hall et al (1994) remark, these findings are a slender basis on which to draw conclusions about the effect of cannabis on schizophrenic symptoms. Until further prospective studies have been carried out, it would be prudent to regard cannabis as a vulnerability factor in relation to major mental illness and to caution at-risk individuals against using the drug.

\section{Cannabis as risk factor for mental illness}

There is no evidence that cannabis is a causal factor in schizophrenia and it is more relevant to consider whether the misuse of the drug constitutes a risk factor for this illness. Supporting evidence is found in a prospective study by Andreasson et al (1987) of 45570 Swedish conscripts, of whom $9.4 \%$ had used cannabis and $1.7 \%$ were 'high consumers' having used more than 50 times. Fifteen-year follow-up data were drawn from national registers of deaths and psychiatric cases. Compared with non-users, the relative risk of schizophrenia was 2.4 in the group that reported use of cannabis at least once, rising to 6.0 among heavy users. Nearly half (430/730) of these high consumers had a psychiatric diagnosis other than psychosis on conscription; controlling for this reduced the relative risk to 2.9. The authors suggest that cannabis consumption is a 'life-event stressor' for individuals vulnerable to schizophrenia. Hall et al (1994) offer a number of alternative explanations. There is a large temporal gap between self-reported cannabis use on conscription and the development of schizophrenia over 15 years, and no data as to whether the cannabis use continued during this time. Drugs other than cannabis could have been taken at any time after conscription.

It should also be noted that as only 49 of the 274 conscripts with schizophrenia had ever tried cannabis, then this drug may only be relevant to a minority of cases. Furthermore, Jablensky et al (1992) demonstrate a striking uniformity in the incidence of schizophrenia in cultures with very different rates of cannabis consumption.

The possibility of a genetic explanation for the association between cannabis use and schizophrenia was raised by McGuire et al (1994). In this study, 23 patients with psychosis and with cannabis in their urine were gender-matched with 46 drug-free controls with psychosis, and the lifetime risk of psychiatric disorder among all the first-degree relatives was ascertained. The cannabis-positive subjects had a significantly greater $(7.1 \%)$ familial risk of schizophrenia than controls $(0.7 \%)$, suggesting that the development or recurrence of acute psychosis in the context of cannabis use may be associated with a genetic predisposition to schizophrenia.

\section{CANNABIS DEPENDENCE}

\section{Evidence for cannabis dependence}

It had been believed that cannabis use did not lead to tolerance and that there was no withdrawal syndrome. However, since the mid-1970s, these views have been challenged by many experimental and observational studies. For example, Jones \& Benowitz (1976) administered oral THC in doses of 70-210 mg/day to subjects for 30 days and noted a progressive loss of the subjective 'high'. This finding was replicated by Georgotas \& Zeidenberg (1979), who gave an average daily dose of $210 \mathrm{mg}$ THC to volunteers for a 4-week period the subjects then "found that the marijuana was much weaker". Withdrawal signs were also found: during the first week of abstinence the subjects "became very irritable, uncooperative, resistant and at times hostile"; they also became hungry and experienced insomnia. These effects waned over 3 weeks. Cessation of smoked cannabis has also been shown to lead to withdrawal symptoms (Haney et al, 1999). The cannabis-withdrawal syndrome has now been unequivocally demonstrated and includes restlessness, anxiety, dysphoria, irritability, insomnia, anorexia, muscle tremor, increased reflexes and autonomic effects including changes in heart rate, blood pressure, sweating and diarrhoea. The syndrome may appear in about 10 hours, and peaks at about 48 hours (Mendelson et al, 1984).

\section{The validity of cannabis dependence}

The Diagnostic and Statistical Manual of Mental Disorders (DSM-IV; American Psychiatric Association, 1994) presents criteria for the diagnosis of psychoactive substance dependence, based largely on the concept of the dependence syndrome (Edwards et al, 1981). The key features of DSM-IV substance dependence are cognitive, behavioural and physiological symptoms, indicating that the individual continues to use the substance despite significant substance-related problems. The criteria include tolerance, a withdrawal syndrome, difficulty in controlling consumption and a pattern of use which leads to a reduction in other important activities. In an empirical study, Morgenstern et al (1994) found the DSM concept of cannabis dependence as least as valid as those for dependence on alcohol, opiates, stimulants and sedatives.

\section{Prevalence and course of cannabis dependence}

From ECA data, Anthony \& Helzer (1991) showed that men had a higher prevalence $(7.7 \%)$ of cannabis abuse or dependence than women $(4.8 \%)$. This was largely due to the greater exposure to illicit drugs of men, since the prevalence of a diagnosis of abuse/dependence among those who had used cannabis more than five times was the same in men and women $(21 \%$ and 19\%, respectively). Extrapolating from these data, Hall et al (1994) suggest that about $17 \%$ of those who used cannabis more than five times would meet DSM-III criteria for dependence, and that for those who have ever used there is approximately a $1 / 10$ risk.

From a New Zealand birth cohort of 1265 children, Fergusson \& Horwood (2000) found that by the age of 21 , nearly $70 \%$ had used cannabis and over $9 \%$ met DSM-IV criteria for cannabis dependence. Key predictors were male gender, ethnic 
minority status and measures of adolescent risk-taking behaviours, including cigarette smoking, conduct problems and a delinquent peer group.

Wiesbeck et al (1996) set out to determine the prevalence of the cannabiswithdrawal syndrome in people who had used the drug but who were not in treatment. In a cohort of 5611 individuals, $31 \%$ had taken the drug on more than 21 occasions in a year. Among these more frequent users, $16 \%$ met criteria for a cannabis-withdrawal syndrome-i.e. at least any one of the following: feeling nervous or irritable, insomnia, tremor, sweats, nausea, gastrointestinal disturbance or appetite change. These individuals had used the drug almost daily for an average of 70 months and even when use of alcohol and other drugs was considered, cannabis use was still significantly related to a selfreport of a history of cannabis withdrawal.

Thomas (1996) found that $35 \%$ of cannabis users said that they could not stop when they wanted to, $24 \%$ continued to use despite problems attributed to the drug and $13 \%$ felt that they could not control their consumption. Restlessness or irritability if they could not use cannabis was reported by $20 \%$ of those surveyed. Interestingly, dependent users were no more likely to report panic or psychotic episodes than those classed as non-dependent. With regard to untoward social consequences, $14 \%$ of cannabis users agreed that the consumption of the drug had caused them to neglect activities previously considered important or enjoyable. These findings (Thomas, 1996) have to be qualified by the low overall response rate of $35 \%$, the use of unvalidated criteria for cannabis dependence and by the lack of data on misuse of alcohol or other drugs among the sample.

Swift et al (1998) interviewed a sample from New South Wales of 243 long-term cannabis users who were smoking 3-4 times a week. A lifetime prevalence of $57 \%$ was found for both DSM-III-R and ICD-10 (World Health Organization, 1992) dependence, but only a quarter perceived that they had a cannabis problem.

\section{VULNERABILITY TO ADVERSE EFFECTS OF CANNABIS}

It has previously been emphasised that constitutional factors such as relative youthfulness, personality and misuse of other drugs, may act as vulnerability factors to the adverse mental effects of cannabis. Mental illness as a vulnerability factor has been reviewed in the previous section.

\section{Adolescence}

There are a number of reasons why adolescence may be regarded as a time of vulnerability for the adverse mental effects of cannabis. First, adolescents may experience emotional problems that cue cannabis use, and their relative youth may lead to an increased risk of adverse mental states on using the drug. Second, regular use of cannabis may interfere with learning and personal development. Last, early initiation of cannabis use may predict an increased risk of escalation in risk and progression to other drugs.

With regard to the possible impact of emotional problems, Newcombe $\&$ Bentler (1988) found a strong relationship between adolescent drug use and the experience of emotional distress, depression and lack of a sense of purpose in life. As to the prospect of adverse mental states on using high doses of cannabis, this review has demonstrated dose-related effects in adults and the younger user is not likely to be at any lesser risk. Crowley et al (1998) found that for adolescents with conduct problems, cannabis use was not benign in that misuse was associated with high rates of dependence and withdrawal.

The possible effects of cannabis consumption on the educational performance of adolescents are not easy to demonstrate in population studies (Hall et al, 1994). Newcombe \& Bentler (1988), having controlled for the higher nonconformity and the lower academic potential among adolescent drug users, found only a modest negative link between drug use and college involvement. Schwartz et al (1989) found short-term memory impairment in 10 cannabis-dependent adolescents compared with matched controls. Test results tended to improve over 6 weeks, which suggested that the deficits observed were due to past cannabis use.

\section{Polydrug use}

A substantial number of young people in the community use a range of drugs which includes cannabis. Ramsay \& Percy (1996) found that $4 \%$ of a group of 16 to 29-year-olds admitted using cannabis and other drugs in the past month, by contrast with $8 \%$ who had used only cannabis. Clinical observation suggests that cannabis users who also misuse other drugs or alcohol seem to experience more severe mental health problems than those who solely take cannabis, but there do not appear to be any substantial published studies on this issue. Polydrug use is a recognised concern in psychiatric populations: for example, Baigent et al (1995) found that $20 \%$ of their dual-diagnosis \#patients misused more than one substance.

\section{Personality}

Given the heterogeneity of the population of cannabis users, it is not surprising that no single personality type or disorder is particular to users of that drug or, indeed, to users of any illicit drug (Allen \& Frances, 1986). However, it is a matter of clinical observation that the use of cannabis by some individuals seems to be predisposed by traits such as social anxiety, anxiety or dysphoria. Such posited use as a form of self-medication to relieve unwanted affects or feelings was not corroborated in a study of cannabis-dependent individuals (Greene et al, 1993). There is good evidence for the comorbidity of drug misuse and some personality disorders. For example, Regier et al (1990) report that some form of substance abuse was identified in $83.6 \%$ of individuals with antisocial personality disorder (ASPD), with an odds ratio of 29.6. It should be appreciated that this very high rate arises because substance abuse is one of the major diagnostic criteria for ASPD; only $16 \%$ of individuals with ASPD did not have a history of substance abuse. The same study showed that the lifetime prevalence of ASPD in cannabis abuse or dependence was $14.7 \%$ with an odds ratio of 8.3. The interaction between ASPD and cannabis use is too complex to explore at length in this review, but it is probable that each disorder exacerbates the adverse effects of the other. See Dolan \& Coid (1993) for a discussion of factors determining outcome in ASPD.

\section{Implications for mental health care}

How should mental health services respond to these findings? The key priorities are: (a) risk-management and care-planning have to be informed by a thorough substancemisuse assessment (Johns, 1997); (b) community and in-patient psychiatric services should develop policies on substance use which balance the treatment needs of 
individual patients with duties of care to other patients and to the general public; and $(c)$ research is needed into treatment interventions for patients with mental illness and substance misuse problems.

\section{REFERENCES}

Allen, M. H. \& Frances, R. (1986) Varieties of psychopathology found in patients with addictive disorders: a review. In Psychopathology and Addictive Disorders (ed. R. Meyer), pp. 17-38. London: Guilford Press.

American Psychiatric Association (1980) Diagnostic and Statistical Manual of Mental Disorders (3rd edn) (DSM-III). Washington, DC: APA.

- (1994) Diagnostic and Statistical Manual of Mental Disorders (4th edn) (DSM-IV). Washington, DC: APA

Andreasson, S., Allebeck, P., Engstrom, A., et al (1987) Cannabis and schizophrenia: a longitudinal study of Swedish conscripts. Lancet, ii, 1483-1486.

Anthony, J. C. \& Helzer, J. E. (199I) Syndromes of drug abuse and dependence. In Psychiatric Disorders in America (eds L. N. Robins \& D. A. Regier). New York: Free Press, Macmillan.

\section{Arndt, S., Tyrrell, G., Flaum, M., et al (1992)} Comorbidity of substance abuse and schizophrenia: the role of pre-morbid adjustment. Psychological Medicine, 22, 379-388.

Ashton, C. H. (200I) Pharmacology and effects of cannabis: a brief review. British Journal of Psychiatry, 178, $101-106$

Baigent, M., Holme, G. \& Hafner, R. J. (1995) Self reports of the interaction between substance abuse and schizophrenia. Australian and New Zealand journal of Psychiatry, 29, 69-74.

Beautrais, A. L., Joyce, P. R. \& Mulder, R.T. (1999) Cannabis abuse and serious suicide attempts. Addiction 94, II55- 1164.

Cantwell, R., Brewin, J., Glazebrook, C., et al (1999) Prevalence of substance misuse in first-episode psychosis. British Journal of Psychiatry, 174, 150-153.

Carney, M. W. P., Bacelle, L. \& Robinson, B. (1984) Psychosis after cannabis abuse. British Medical Journal, 288, 1047

Chaudry, H. R., Moss, H. B., Bashir, A., et al (199I) Cannabis psychosis following bhang ingestion. British Journal of Addiction, 86, 1075-1081.

Chopra, G. S. \& Smith, J.W. (1974) Psychotic reactions following cannabis use in East Indians. Archives of General Psychiatry, 30, 24-27.

Cleghorn, J. M., Kaplan, R. D., Szechtman, B., et al (1991) Substance abuse and schizophrenia: effect on symptoms but not on neurocognitive function. Journal of Clinical Psychiatry, 52, 26-30.

Crowley, T. J., Macdonald, M. J., Whitmore, E. A., et al (1998) Cannabis dependence, withdrawal and reinforcing effects among adolescents with conduct symptoms and substance use disorders. Drug and Alcohol Dependence, 50, 27-37.

Dixon, L., Haas, G., Wedien, P. J., et al (1990) Acute effects of drug abuse in schizophrenic patients: clinical observations and patients' self-reports. Schizophrenia Bulletin, 16, 69-79.

Dolan, B. \& Coid, J. (1993) Psychopathic and Antisocia Personality Disorders: Treatment and Research Issues. London: Gaskell.

\section{CLINICAL IMPLICATIONS}

- Among those who have ever taken cannabis, $\mathrm{I} / \mathrm{I0}$ are at risk of dependence.

- Heavy cannabis misuse leads to the risk of psychotic episodes, and aggravates the symptoms and course of schizophrenia.

- For any psychiatric patient, risk-management and care-planning is incomplete without a thorough assessment of substance misuse.

\section{LIMITATIONS}

- The available literature shows a preponderance of case reports and uncontrolled studies.

- Epidemiological findings from one setting cannot be assumed to generalise to other cultural groups.

- It is not easy to determine causal explanations from the studies cited.

ANDREW JOHNS, FRCPsych, Department of Forensic Psychiatry, Institute of Psychiatry, De Crespigny Park, Denmark Hill, London SE5 8AF. Tel: 02079193123

(First received 22 July 1999, final revision 4 September 2000, accepted 6 September 2000)

Edwards, G., Arif, A. \& Hodgson, R. (198I)

Nomenclature and classification of drug-and alcohol-

related problems: a WHO memorandum. Bulletin of the World Health Organization, 59, 225-242.

Fergusson, D. M. \& Horwood, L. J. (2000) Cannabis use and dependence in a New Zealand birth cohort. New Zealand Medical Journal, II3, 56-58.

Georgotas, A. \& Zeidenberg, P. (1979) Observations on the effects of four weeks of heavy marijuana smoking on group interaction and individual behavior. Comprehensive Psychiatry, 20, 427-432.

Ghodse, A. H. (1986) Cannabis psychosis. British Journal of Addiction, 8I, 473-478.

Greene, R. L., Adyanthaya, A. E., Morse, R. M., et al (1993) Personality variables in cocaine and marijuana dependent patients. Journal of Personality Assessment, $6 \mathbf{I}$ 224-230

Hall, W., Solowij, N. \& Lemon, J. (1994) The Health and Social Consequences of Cannabis Use. Monograph Series No. 25. Canberra: Australian Government Publishing Service.

Haney, M., Ward, A. S., Comer, S. D., et al (1999) Abstinence symptoms following smoked marijuana in humans. Psychopharmacology (Berl), I4I 395-404.

\section{Jablensky, A., Sartorius, N., Ernberg, G., et al} (1992) Schizophrenia: manifestations, incidence and course in different cultures. Psychological Medicine. Monograph Supplement 20.

Johns, A. (1997) Substance misuse: a primary risk and major problem of comorbidity. International Journal of Psychiatry, 9, 233-241.

Jones, R.T. \& Benowitz, N. (1976) The 30-day trip Clinical studies of cannabis tolerance and dependence. In Pharmacology of Marijuana, Vol. 2 (eds M. C. Braude \& S. Szara). New York: Academic Press.
Keshaven, M. S. \& Lishman, W. A. (1986) Prolonged depersonalization following cannabis abuse. British Journal of Addiction, 81, 140-142.

Linszman, D. H., Dingemans, P. M. \& Lenior, M. E. (1994) Cannabis abuse and the course of recent-onset schizophrenic disorders. Archives of General Psychiatry 5I, 273-279.

Lishman, W. A. (1998) Organic Psychiatry: The Psychological Consequences of Cerebral Disorder (3rd edn). Oxford: Blackwell.

Lukoff, D., Lieberman, R. \& Neuchterlein, K. (1986) Symptom monitoring in the rehabilitation of schizophrenic patients. Schizophrenia Bulletin, 12, 578 603.

McGuire, P. K., Jones, P., Harvey, I., et al (1994) Cannabis and acute psychosis. Schizophrenia Research, 13 |61-167.

Mathers, D. C. \& Ghodse, A. H. (1992) Cannabis and psychotic illness. British Journal of Psychiatry, 16I 648-653.

Mendelson, J. H., Mello, N. K., Lex, B.W., et al (1984) Marijuana withdrawal syndrome in a woman. American Journal of Psychiatry, 14I, 1289-1290.

Menezes, P. R., Johnson, S., Thornicroft, G., et al (1996) Drug and alcohol problems among individuals with severe mental illness in south London. British Journal of Psychiatry, 168, 612-619.

Morgenstern, J., Langenbucher, J. \& Labouvie, E.W. (1994) The generalizability of the dependence syndrome across substances: an examination of some properties of the proposed DSM-IV dependence criteria. Addiction, 89, $1105-1113$.

Negrete, J. C., Knapp, W. P., Douglas, D. E., et al (1986) Cannabis affects the severity of schizophrenic symptoms: results of a clinical survey. Psychological Medicine, 16, 515-520 
Newcombe, M. D. \& Bentler, P. (1988) Consequences of Adolescent Drug Use: Impact on the Lives of Young Adults. Newbury Park, CA: Sage.

Peralta, V. \& Cuesta, M. J. (1992) Influence of cannabis abuse on schizophrenic psychopathology. Acta Psychiatrica Scandinavica, 85, 127-130.

Ramsay, M. \& Percy, A. (1996) Drug Misuse Declared Results of the 1994 British Crime Survey. London: Home Office.

Regier, D. A., Farmer, M. E., Rae, D. S., et al (1990) Comorbidity of mental disorders with alcohol and other drug abuse. Results from the Epidemiologic Catchment Area (ECA) study. Journal of the American Medical Association, 264, 25II-2518.

Reilly, D., Didcott, R., Swift, W., et al (1998) Longterm cannabis use: characteristics of users in Australian rural areas. Addiction, 93, 837-846.

Rottanburg, D. R., Robins, A. H., Ben-Arie, O., et a (1982) Cannabis-associated psychosis with hypomanic features. Lancet, ii, 1364-1366.

Schwartz, R. H., Gruenewald, P. J., Klitzner, M., et ol (1989) Short-term memory impairment in cannabisdependent adolescents. American Journal of Disorders of Childhood, 143, 1214-1219.
Swanson, J.W., Holzer, C. E. 3rd, Ganju, V. K., et al (1990) Violence and psychiatric disorder in the community: evidence from the Epidemiologic Catchment Area surveys. Hospital and Community Psychiatry, 4I, 76I-770.

Swift, W., Hall, W., Didcott, P., et al (1998) Patterns and correlates of cannabis dependence among long-term users in an Australian rural area. Addiction, 93, |149-1160.

Szymanski, H. V. (198I) Prolonged depersonalisation after marijuana use. American Journal of Psychiatry, $\mathbf{1 3 8}$ $23 \mid-233$.

Talbott, J. A. \& Teague, J.W. (1969) Marijuana psychosis. Journal of the American Medical Association, 210, 299-302.

Tennant, F. S. \& Groesbeck, C. J. (1972) Psychiatric effects of hashish. Archives of General Psychiatry, 27, 133-136.

Thacore, V. R. \& Shukla, S. R. P. (1976) Cannabis psychosis and paranoid schizophrenia. Archives of General Psychiatry, 33, 383-386.

Thomas, H. (1993) Psychiatric symptoms in cannabis users. British Journal of Psychiatry, 163, 141-149.
- (1996) A community survey of adverse effects of cannabis use. Drug and Alcohol Dependence, 42 20I-207.

Thornicroft, G. (1990) Cannabis and psychosis. Is there epidemiological evidence for an association? British Journal of Psychiatry, 157, 25-33.

Troisi, A., Pasini, A., Saracco, M., et al (1998) Psychiatric symptoms in male cannabis users not using other illicit drugs. Addiction, 93, 487-492.

Tsuang, M. T., Simpson, J. C. \& Kronfol, Z. (1982) Subtypes of drug abuse with psychosis. Archives of General Psychiatry, 39. 14I-147.

Wiesbeck, G. A., Schuckit, M. A., Kalmijn, J. A., et al (1996) An evaluation of the history of a marijuana withdrawal syndrome in a large population. Addiction, $9 \mathbf{l}$ 1469-1478.

Wing, J. K., Cooper, J. E. \& Sartorius, N. (1974) Measurement and Classification of Psychiatric Symptoms: An Instruction Manual for the PSE and CATEGO Program. Cambridge: Cambridge University Press.

World Health Organization (1992) The ICD-10 Classification of Mental and Behavioural Disorders. Geneva: WHO 